Témoigner Témoigner. Entre histoire et mémoire

Getuigen Revue pluridisciplinaire de la Fondation Auschwitz

$121 \mid 2015$

Violences radicales en scène

\title{
La Figure des Tiers dans le processus mémoriel
}

Une conversation entre Gilbert Ndahayo et Alexandre Dauge-Roth

Alexandre Dauge-Roth

(2) OpenEdition

Journals

Édition électronique

URL : https://journals.openedition.org/temoigner/3389

DOI : 10.4000/temoigner.3389

ISSN : 2506-6390

Éditeur :

Éditions du Centre d'études et de documentation Mémoire d'Auschwitz, Éditions Kimé

Édition imprimée

Date de publication : 1 octobre 2015

Pagination : 108-120

ISSN : 2031-4183

Référence électronique

Alexandre Dauge-Roth, «La Figure des Tiers dans le processus mémoriel », Témoigner. Entre histoire et mémoire [En ligne], 121 | 2015, mis en ligne le 01 octobre 2016, consulté le 07 février 2022. URL: http://journals.openedition.org/temoigner/3389 ; DOI : https://doi.org/10.4000/temoigner.3389 


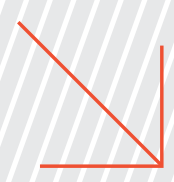

$\rightarrow$ Par Alexandre

Dauge-Roth, Bates College

\section{La Figure des Tiers dans le processus mémoriel Une conversation entre Gilbert Ndahayo et Alexandre Dauge-Roth}

Alexandre Dauge-Roth : Comme point de départ de notre conversation, nous pourrions partir de la question suivante : est-ce que le tiers a un rôle à jouer dans ta démarche autodocumentaire, quant aux possibilités de dire ou de raconter? Autrement dit quelle est la place de différents interlocuteurs tiers dans tes documentaires et ta démarche filmique ? Si le génocide met face-à-face la victime et le bourreau ainsi qu'un tiers-acteur témoin, dans l'après-génocide cela se complique puisque viennent s'ajouter les figures propres à l’adresse mémorielle : le tiers-destinataire (spectateur ou lecteur), le tiers-témoin celui qui se constitue en tiers pour passer son message et survivre tout comme ces autres tiers posthumes que sont les absents, les disparus, ceux et celles pour lesquels la mémoire se construit, figures tierces de la relation qu'entretiennent les vivants entre eux. En réfléchissant sur cette problématique au sein de la démarche testimoniale, j’ai été amené à distinguer quatre figures du tiers.

La première figure du tiers est tout d'abord un tiers intérieur que le survivant doit faire advenir en lui et qui lui offre la possibilité de créer un dialogue intérieur avec soi-même. Il faut construire une sorte de «tu », ce double de soi-même, avec lequel le « je » peut dialoguer. Dans l'autodocumentaire, ce tiers intérieur renvoie aux scènes où toi-même tu es à la fois personnage et réalisateur du documentaire. En somme, tu mets en place un dispositif où toi comme survivant cherche à établir un dialogue avec ton tiers intérieur.

À ce tiers intérieur s'oppose un tiers extérieur. Les figures du tiers extérieur, elles, sont plurielles. Il peut s'agir à la fois des survivants ou d'autres personnes à qui tu t'adresses, ils peuvent être des Rwandais, comme toi, ou des Occidentaux, comme moi, qui cherchent à savoir ce qui s'est passé et à définir leur rôle vis-à-vis des survivants. Ces tiers sont l'ensemble des personnes auprès de qui tu recherches 


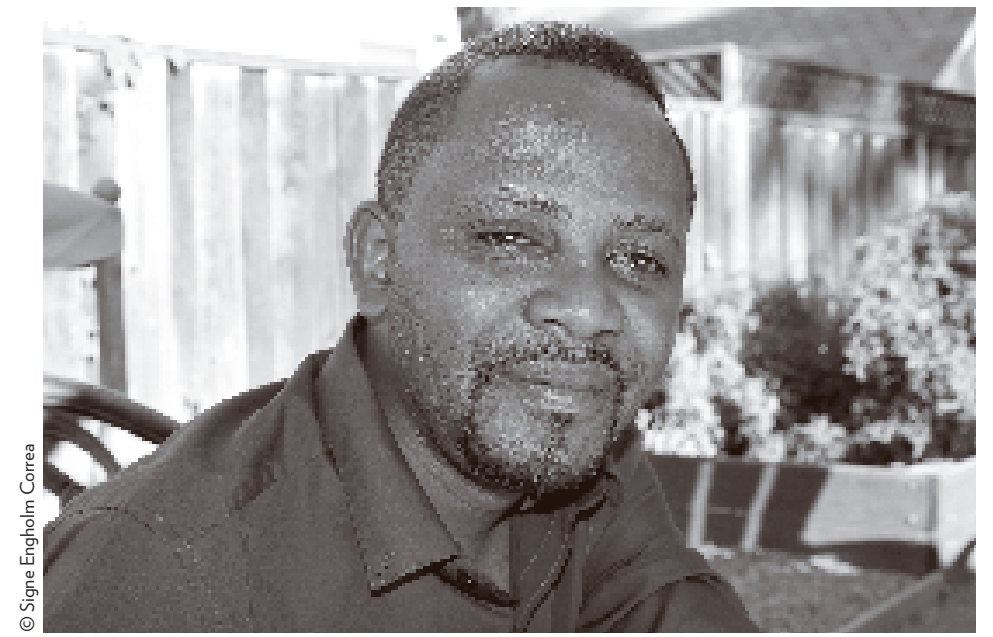

_ Gilbert Ndahayo.

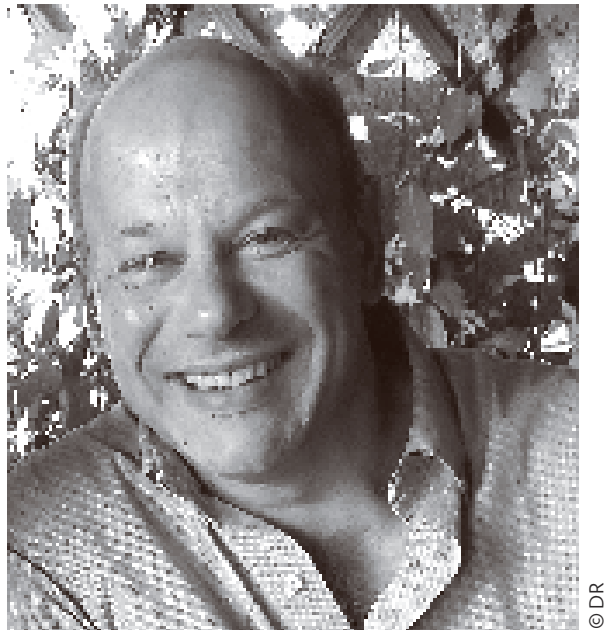

- Alexandre Dauge-Roth.

une certaine forme d'écoute, une certaine réponse par rapport à l'expérience du génocide dont tu témoignes. Voilà qui constituerait une première paire de tiers (tiers intérieur et tiers extérieur) qui relève d’une dynamique complémentaire, l'un nourrissant l'autre et inversement.

Ensuite, il y a deux tiers qui, eux, sont définis par leur rapport temporel à l'énonciation testimoniale. Le premier tiers est le tiers posthume qui renvoie au dialogue que toi, survivant, tu établis avec les morts. De ce tiers, tu te fais à la fois le mémorialiste et l'archiviste en œuvrant à entretenir son actualité à travers un devoir de mémoire et un travail sur la mémoire. Héritier d'un passé que tu n'as pas choisi, il n'en demeure pas moins essentiel que tu te positionnes en tant qu'héritier vis-à-vis de ces tiers posthumes.

Finalement, il y a le tiers à venir. En somme un tiers qui n'existe pas encore et qui serait un Gilbert Ndahayo à venir, celui que le processus de l'entreprise testimoniale ou le processus du film fait advenir. C'est un tiers auquel le survivant aspire consciemment ou non au sein même de son témoignage. Ce tiers signale avant tout la possibilité de pouvoir se projeter dans l'avenir, ce qui n'est pas un geste évident pour un survivant pour qui demeure toujours le risque d'être pétrifié ou prisonnier d'un passé traumatique. D'où l'importance que le dialogue avec les morts soit mis en interaction avec cet autre dialogue que le témoin entretient avec un tiers à venir.

Qu'est-ce que tu penses de ces quatre figures de tiers? Est-ce que certaines sont pertinentes à tes yeux et jouent un rôle dans ta démarche? Est-ce que certaines de ces figures n'ont aucune pertinence? Penses-tu que ces quatre formes de dialogue à travers ces quatre figures du tiers sont une entrée possible pour comprendre l'agencement qui est au fondement de ton autodocumentaire Rwanda:Beyond the Deadly Pit? 
Gilbert Ndahayo : J'ai une question avant de te répondre. Est-ce qu'il existe un tiers en transition entre ces quatre tiers? Une transition à envisager dans le temps ou dans l'espace. Intérieur ou extérieur, c'est l'espace. Posthume ou à venir, c'est le temps. Est-ce qu'ily a un tiers au milieu? Donc, une personne qui veut bien avancer, mais qui est toujours coincée dans le passé. Et je te pose cette question parce que là où je suis maintenant, dans tous mes films, il y a toujours la même fin, une fin ouverte. C'est ouvert pour l'avenir, mais le trajet, lui, il n'est pas complet. Lors de la discussion du premier visionnement de The Rwandan Night, le documentaire qui suit Rwanda: Beyond the Deadly Pit, un survivant qui a fait trois heures de voiture pour venir voir le film a dit à l'auditoire en enlevant sa chemise pour montrer ses cicatrices : « Je suis un survivant du génocide. » Si l’on parle de blessures du génocide, elles ne peuvent pas guérir, elles demeurent des cicatrices indélébiles. Comment est-ce que l'on peut envisager ce tiers en transition?

A.D.-R. : Je dirais que cette transition renvoie au tiers à venir, parce que l'idée du tiers à venir représente une instance qui est autre que celui que tu es présentement. Si ta personne est en transition, c'est qu'elle aspire à ce tiers à venir et qu'elle n'est donc pas seulement en dialogue avec les tiers du passé. Au centre de ces quatre postures du tiers, il y a le « je » du sujet témoignant. Effectivement, comme tu le dis, ce n'est pas un « je » qui est statique ou défini une fois pour toutes par son passé, mais qui, justement, à cause du tiers à venir s'envisage comme ouvert à la transition, l'appelant même dans plus d'un cas de ses vœux. Un survivant du génocide aspire à une forme de renouveau. Il œuvre à s'envisager comme étant en devenir, mais il n’y a jamais de garantie. En ce sens, le tiers à venir est certes un personnage désiré, fictionnel, voire utopique, mais c'est une figure essentielle, car elle inscrit le sujet témoignant dans une dynamique transitionnelle.

Peut-être, commençons avec le tiers intérieur dans Rwanda: Beyond the Deadly Pit où tu te filmes et où tu opères un travail de montage qui a duré plus de quatre ans. Est-ce que cette longue genèse où tu as travaillé à agencer les voix des autres tout comme la tienne de manière dialogique participe à la construction de ce dialogue intérieur? Ce désir d'un montage polyphonique vise-t-il à prendre un peu de distance vis-à-vis de soi-même? Le tiers intérieur est-il une possibilité de s'envisager autre, soi-même comme un autre?

G. N. : Je suis tenté de dire que le «tiers » fait référence à «l'autre», une personne qui n'est pas moi. Mais en réalité, si je réfléchis à moi-même, je pense à « l'autre » dont les jugements et les actions se projettent en moi sous la forme d'un raisonnement et d'un entendement. Le regard d'une caméra est unidimensionnel de par son objectif. Dans l'autodocumentaire, un cinéaste se filme en filmant les « autres ». Le film tourne autour du cinéaste qui s'expose pour montrer son tiers. Donc, le cinéaste devient un protagoniste de son propre film. Ainsi le film est plus intime et l'intensité de l'image de soi que l'on filme donne accès à une pluralité de tiers.

Pour mieux apprécier toute démarche autodocumentaire, il faut prendre en 
considération la situation sur le terrain du tournage. Dans le cas de Rwanda: Beyond the Deadly Pit, le processus pour créer des images était plutôt agressif. Je ne répondais qu’à moi-même. Les survivants me disaient d'arrêter de filmer ici et de filmer plutôt là-bas, mais je suivais mon idée directrice. Le processus de filmer obéissait pour moi à un impératif : «je dois avoir ces images » et aucun auteur-réalisateur ne se laissera dicter ses choix par un tiers. Mon tournage était donc conditionné par le temps, une sorte de course contre la montre. Sans ces images, je savais que je n'aurais aucune image du et pour le tiers extérieur des événements qui se déroulaient devant mes yeux et le champ visuel de ma caméra. Par exemple, dans le cas de l'ouverture de la fosse où se trouvaient mes parents, les corps y étaient maintenant et demain ils n'y seraient plus. Je me disais que je devais avoir les images des survivants dans leurs univers, à savoir les endroits mêmes où les crimes de génocide ont été commis. L’idée non-scriptée au départ était le retour d’un survivant du génocide pour « parler » et au centre du projet filmique, il y avait la fosse mortelle pour laquelle aucune image n'existait parce que personne n'avait encore ouvert cette fosse. Cette idée a servi de points de départ aux images de déterrement de mes parents. Ensuite, une idée en a engendré une autre. Le déterrement des personnes qui ont été jetées dans la fosse « mortelle » a obligé les survivants à laver leurs ossements, à préparer les corps pour les déposer dans des cercueils et faire une prière avant de les amener au Stade régional de Nyamirambo et, finalement, au site mémorial de Gisozi. Ces pratiques génèrent des images où les survivants se trouvent comme prisonniers du passé. La plupart d'entre eux étaient avec les morts. À ce moment-là, c'est un passé que l'on déterre, mais aussi le même passé qui est toujours présent en eux. Le processus du tournage c'est une action qui représente le tiers posthume tout comme certains tiers extérieurs dans leurs liens avec les morts.

Le processus de montage, c'est là où je suis seul avec le passé devant moi, avec le matériel quej’ai tourné. Là, c'est différent. L’agressivité n'est plus là. La course contre la montre de documenter le passé à travers le présent de l'objectif est terminée. Je ne me bats plus contre la montre, mais plutôt contre et pour moi-même. Je suis l'image que je crée de moi et des autres. Ces images doivent être convaincantes. Et pour me convaincre, je dois maintenant me poser et dire « voilà, il faut recréer le passé », mais le passé n'est plus là et les corps des morts sont partis. On dit aux survivants que « là où les victimes du génocide sont, elles sont enterrées en dignité. » Donc les activités d'exhumation, toute la culture de la commémoration, les lieux où les morts doivent avoir le respect et le repos éternel n'existent plus. Ça existe seulement dans les images que j’ai créées. Ces images, que veulent-elles dire en définitive? Ces images en soi, elles sont violentes certes, parce que je les ai filmées avec cette agressivité, cette pression d'avoir ces images. La caméra est là sur l'épaule. Il y a le poids de la caméra, le poids du passé, mais il y a aussi le poids du temps qui file, car si tu ne prends pas ces images, elles n'existeront jamais.

Le processus de montage lui n'est pas agressif parce que là tu es seul, confronté avec ce que tu vois qui ne te répond pas. La réponse, qui t’appartient, elle est d’abord émotionnelle. De quoi est-ce que la personne témoigne, qu'est-ce qu'elle dit? Que 
disent les gens qui l'écoutent? Est-ce que ça suscite des émotions? Qu'est ce qu’ils font avant de parler ? Il y a des gens qui allument des bougies, il y en a d'autres qui sont directement affectés par le témoignage et qui pleurent.

Moi, dans mon agressivité, je dois toujours capter les réactions que suscite le témoignage du survivant parmi ses interlocuteurs, ces tiers extérieurs que je filme. Au montage, ma responsabilité est de produire une archive qui va attester dans la longue durée qu’à telle date tel événement a eu lieu. Quand une personne dit dans une séquence : « aujourd'hui nous commémorons le dix-septième anniversaire du génocide », plusieurs temporalités sont en jeu, il nous faut remonter dans le temps. Nous sommes en 2013, j'ai filmé en 2006 et cette personne raconte, au moment de la projection, ce qui s'est passé dix-sept ans plus tôt, en 1994. La responsabilité d'un cinéaste contemporain est donc de créer, pour les spectateurs, les conditions d'une réponse qui ne peut qu'être différée, mais qui toutefois engage son présent.

Je commence à produire le film en 2007, mais il sort en 2013. Un des défis est de capturer l'archive dans la mémoire du survivant qui témoigne d'un passé qui est lié au génocide - comme les premiers actes de génocide de 1959 - mais pour lequel il n'y a pas d'archives hormis dans la tête du survivant. Visuellement, les images de ce passé antérieur font donc défaut en dépit du fait qu'elles informent l'image présente. Les images de 1959 sont dans la tête, elles ont été mémorisées par les personnes qui parlent ici. Maintenant, je dois produire des séquences qui donnent sens au passé dans le présent du montage. La question qui se pose après le recueil du témoignage est de savoir qu'est-ce qui s'est passé après 1994 dans la vie du témoin de ce génocide. Que s'est-il passé depuis et qui justement vient influencer la manière dont ce témoin se positionne vis-à-vis du passé et en rend compte. Cette absence de coïncidence entre l'événement dont le témoin parle, le présent d'où il en parle, le moment du montage et enfin le temps de la projection du film génère donc une multitude de tensions et d'écarts qui altèrent la médiation du souvenir des témoins lors de leur prise de parole devant la caméra. Cette non-coïncidence entre l'événement tel qu'il s'est produit, son souvenir tel que le relatent les témoins plusieurs années plus tard et les choix du cinéaste d'en rendre compte en différé plusieurs années après à travers des manipulations techniques, ont des incidences immédiates sur le ressenti des tiers et sur ce que comprendront les spectateurs.

Face à cette question du différé et de l'absence de coïncidence, je me positionne selon trois axes.

Le premier axe renvoie au point de vue du gouvernement, à la façon dont cette situation est gérée collectivement, c'est-à-dire la politique de la mémoire, de la réconciliation et de la justice. Qu'est-ce que le pays fait pour remédier à la situation psychosociale des survivants? Chaque survivant a sa représentation du génocide qui lui est propre, mais dans les documents officiels et durant les commémorations, on remarque que c'est toujours la même histoire, alors que ceci est impossible. Les souvenirs ne se ressemblent jamais en dépit des généralités. C'est seulement parce que ce sont toujours les mêmes personnes qui sont interviewées pour exprimer les sentiments et souhaits de l'ensemble des survivants que se dégage ce consen- 
sus en forme de trompe-l'œil. Que se passe-t-il au sein du survivant à qui l'on ne donne jamais l'occasion de s'exprimer ? Ce survivant invisible et inaudible, à quoi aspire-t-il en tant qu'individu? Il rentre chez lui, il déterre les siens, il chante, il va à l'église, il commémore, il honore, voilà. Il essaie d'oublier. Il n'est plus chez lui, n’a plus de chez soi, il est ailleurs. Au Rwanda, c'est la politique qui t'assigne ta place et c'est là que tu dois être que tu le veuilles ou non. Est-ce que moi, je veux être là? Est-ce qu'à la personne qui est morte, on lui pose la question de savoir où elle veut être. La survivante, est-ce qu'on lui demande si on doit emmener la personne qui lui est chère ? Ces personnes qui sont mortes : mon père, ma mère et ma sœur; ce sont là mes propriétés. En plus de cela, on ne me demande pas si je dois laver leurs ossements, si je dois les mettre dans un cercueil ou si j’opte pour la crémation. Je pose ces questions que le mort lui ne pose pas.

A.D.-R. : L’enjeu ici c'est que la politique définit les rituels de la mémoire du génocide et que ces rituels ne répondent pas nécessairement à tes attentes, tes questions ou ta vision du devoir de mémoire?

G. N. : La mémoire du génocide est définie par la société. Mais la société c'est qui ? C'est moi et toi. Dans la prise des décisions affectant une société, il y a une voix majoritaire qui décide sans trop se préoccuper des autres voix. Est-ce que je suis content que quelqu'un d'autre, comme toi, aille dans un mémorial et s'arrête devant les vitrines où la personne qui m'est chère se trouve pour être prise en photo ? Et si je ne suis pas content avec ce fait, qu'est-ce que je peux faire? Est-ce que je peux venir te frapper ou te demander de l'argent? Il y a une question centrale ici que doit se poser le tiers extérieur et à laquelle il se doit de répondre avant même de répondre à l'injonction et aux attentes de la politique mémorielle du gouvernement. Est-ce que toi, quand tu vas au Rwanda, est-ce que tu te sens obligé ou sollicité d'aller voir ces sites mémoriaux ? Est-ce qu'il y a des panneaux d'affichage qui t'invitent à commémorer ces histoires de 1994 ? Moi, je veux attirer l'attention du monde en essayant de répondre aux questions qui définissent l'attente des tiers-spectateurs auxquels je m'adresse, qu'ils soient des survivants ou non.

A.D.-R. : Il y a les personnes que tu filmes en 2006, qui elles-mêmes accomplissaient un certain nombre de rituels, parlaient des morts, souscrivaient au devoir de mémoire. Ensuite, il y a ton travail à toi et ta façon d'approcher le devoir de mémoire. Quelle est la relation entre ton travail de cinéaste archivant et les rituels de commémoration que tu as filmés ? S'il y a bien, comme tu l'as dit, d'un côté une politique qui définit les rituels de commémoration et puis, de l'autre, des survivants qui, comme toi, ont leurs propres désirs et formes de commémoration, est-ce que ton travail cherche à leur offrir un lieu de parole où ils peuvent s'exprimer? Quelle est la relation mémorielle que le travail de montage entretient vis-à-vis des rituels de commémoration que tu as filmés? 
G. N. : Le survivant, comme tu le vois dans le documentaire, il dit « Bonjour Gilbert, il faut que toi tu te filmes aussi. » Donc, c'est une invitation, ça veut dire que moi aussi je suis un survivant et je dois faire partie de ces activités collectives de commémoration et que, ou je me filme ou quelqu'un d'autre me filme. En filmant, je suis un individu qui réimprime son devoir. Ma façon de commémorer n’obéit pas à ce qui est défini par la politique. C'est en filmant que j'accomplis mon devoir de mémoire. Cette invitation est une autre façon de dire « venez nous aider en essayant de commémorer.» Même si je suis agressif, je suis toujours l'hôte, le commémorant, le survivant avant d'être le réalisateur du film. Il y a des moments dans le tournage où des membres proches de ma famille me disent : « Gilbert, arrête de filmer : c'est cruel. » C'est une façon de dire «Arrête d'être solitaire, tu es comme nous. » Je me suis isolé d'eux pour les filmer.

Quant à la question du montage, là je l'effectue en fonction des émotions que les images filmées évoquent en moi. Je dois trouver un point de rencontre, des carrefours de survie qui existent entre un jeune survivant, un adulte et une femme, sachant que chacun d'entre eux a des points de vue différents parce qu'ils ont vécu des moments différents soit individuellement soit collectivement. Je crée un agencement de voix pour créer un témoignage qui les organise selon un même fil conducteur. Il faut que le film commence avec un début et ait un milieu. Les trois points de vue - jeune, adulte, femme - doivent être mis en dialogue pour que toutes les voix de ces tiers extérieurs à moi et aux miens qui ne sont plus soient représentées.

Sur le banc de montage, je ne regarde pas les images que j’ai filmées parce que les images de ma caméra ont continué de tourner dans ma tête. Alors, je commence avec le son. Je commence sans le visage de la personne. Je m’imagine qui elle est à travers ce qu'elle dit. Je suis ses émotions. Des fois, la voix est haute, des fois basse. Des fois, c'est le silence ou le cri, cela dépend de l'évolution du témoignage. Si c'est au début, c'est toujours une voix basse qui commence en chuchotant, comme si la voix elle-même avait peur d'être entendue ou que le survivant avait peur de parler. Mais les voix évoluent, car le microphone circule parmi la communauté des survivants ; ce qui fait que les tensions montent.

Au départ, je défigure, j’écoute seulement les voix, etje les monte pour obtenir un premier agencement. À certains moments, je veux entendre un jeune qui était dans cette situation quand il regardait ses parents être massacrés. Puis je veux entendre une femme. Dans les voix quej'ai choisies, il y a trois points de vue exprimant presque la même chose, mais différemment. Le premier survivant dit « j’ai vu... », le second enchaine avec « ils étaient... » et le troisième dit « j’ai entendu... » Pour compléter la séquence, je reprends où j'en étais avec le premier survivant et puis je greffe des autres points de vue. Après le son du témoignage, je mets les visages. J'ai envie de voir qui témoigne. À mes yeux, est-il sincère ? Là, je vois l'expression du regard et le regard, qu'exprime-t-il ? Est-ce qu'il parle avec les gestes ? Est-ce que le souvenir en soi lui donne l'air d'être ramené à l'abattoir ? Oui, il se souvient, il est pétrifié. Oui, il voit la photo de sa mère et les souvenirs reviennent. Il n'en peut plus, il crie. Là, je comprends qu'il est au milieu du site du massacre, un stade ou une église, il 
est avec sa mère quand on l'emmène pour la tuer. La mémoire de ce survivant n'a jamais quitté ce lieu de massacre.

A.D.-R.: Dans le travail de montage aussi, il y a des mises en dialogue que tu fais qui ne sont pas seulement entre différents survivants. Il y a aussi ta voix, toi tu deviens un personnage de ton film et tu te mets en situation de dialogue avec les autres. Il y a cette séquence avec le secrétaire général des Nations Unies Ban Ki-moon, lors de sa visite au Rwanda. Tu le filmes devant les membres du Parlement rwandais dans le bâtiment appelé CND, là où tu as trouvé refuge pendant le génocide. Ban Ki-moon met des écouteurs sur ses oreilles pour comprendre et entendre ce qu'on lui dit. Et ensuite, on te voit, toi aussi, mettre tes écouteurs sur tes oreilles. Quel type de dialogue est-ce que tu essaies de créer dans ce type de montage?

G. N. : Ces deux événements ont été filmés séparément, mais sont liés dans l’unicité d’un lieu. Le bâtiment de l'ancien Conseil national de développement (CND) qui accueille toujours le Parlement rwandais. Pendant le génocide en 1994, je me suis réfugié au CND pour survivre. Je n'étais pas avec mes parents lorsqu'ils ont été tués. Les forces du FPR se sont battues pour nous protéger et arrêter le génocide. Une fois Kigali libéré, je suis sorti des caves du CND pour rentrer chez moi. C'est en voyant notre maison en ruine et en sentant l'odeur des morts que j'ai compris que mes parents n'étaient plus. Je voulais savoir ce qui leur était arrivé, connaître leur sort. Je voulais écouter et comprendre. Mais j’étais seul, assis devant la porte de notre maison en ruine.

Cette séquence est très révélatrice sur moi : mon moi intérieur. Dans Rwanda: Beyond the Deadly Pit, je documente ce qui se passe dans ma vie, chez moi sous laforme d'un journal audio-visuel. Dans ce journal, si on le lit très bien, Ban Ki-moon veut que l'on traduise pour lui ce que c'était un génocide, ou que l'on traduise le kinyarwanda. Mais au lieu de cela, on lui a traduit ce que les Nations Unies n'avaient pas fait entre 1990 et 2007. On lui traduit les réalités de l’après-génocide. Parallèlement, moi, je fais aussi un travail de traduction. Que ce soit le sous-titrage, ou en termes d'écriture du scénario du documentaire, je traduis la réalité de l'après-génocide sur l'écran. Si tu me demandes si j'ai un script du documentaire Rwanda: Beyond the Deadly Pit, je n'en ai jamais eu. Un script aurait limité les champs du tournage. Le documentaire a été écrit et réécrit au montage en suivant mes émotions. Quelle est l'émotion que Ban Ki-moon traduit en mettant ses écouteurs? Quel est l'effet que cela me fait de le voir là, à l'endroit où des gens ont donné leur vie pour ma survie et ont secouru les survivants alors que les Nations Unies ne voulaient pas intervenir?

A.D.-R. : Est-ce que ça veut aussi dire que quelque part tu construis ta voix ou ta traduction en mettant en dialogue le discours de Ban Ki-moon sur le rôle passif des Nations Unies, son acte de contrition au Parlement rwandais et ton discours à toi vis-à-vis du passé ? Est-ce que c'est une façon de signifier aux tiers qu'ils sont en présence de deux discours distincts pour qu'ils se demandent ce que ces deux 
discours sur un «même passé » ont en commun ? Comment est-ce que ces deux discours peuvent s'entendre en somme, entamer un dialogue?

G. N. : Je crois que c'est une bonne remarque. Ban Ki-moon ne se repent ni personnellement ni officiellement. Il évite tout simplement la question en disant qu'en 2005 les Nations Unies ont reconnu la responsabilité de protéger le monde contre les crimes contre l'humanité et d'autres violations des droits de l'homme. Il ajoute même : «It looks like I am sitting in the General Assembly of the United Nations ! » Ces propos qui ont un ton moqueur sont bien reçus par les membres du Parlement rwandais. Là, moi, j'ai envie de le comprendre. Parce que ce qu'il dit, je ne le comprends pas et je ne comprends pas pourquoi les parlementaires rigolent. Il y a toute une série d'informations et d'options que je dois gérer et digérer en même temps. Je mets les écouteurs pour comprendre ce qu'il dit. Cette scène de Ban Ki-moon, signale un peu ma frustration, d'où l'agressivité dans le montage.

A.D.-R. : Est-ce que ce travail de montage entre réalisateur et personnage est produit petit à petit à travers ta double capacitéà, d’abord, répondre émotionnellement aux voix que tu as archivées et, ensuite, à redistribuer ces voix dans un agencement inédit où tu prends en somme la parole à travers celles des autres? Pour pouvoir espérer se réconcilier avec soi-même, est-ce que cela demande de faire ce type de détour par la voix des autres?

G. N. : Je ne crois pas que ce soit ça. En documentant, en tournant et en montant ces images, c'est pour moi plutôt une réconciliation avec les autres. Mais, je me réconcilie avec qui? Il y a des génocidaires qui doivent être punis, il y a des survivants qui parlent, et il y a aussi des étrangers, des bystanders. Je me réconcilie avec eux. En mettant les écouteurs, j'essaie de les écouter sans les voir, de les comprendre. Quand je suis en tournage, les gens me disent souvent : « viens avec nous, tu n'es pas avec nous. »C'est une forme d'offense qui est toutefois acceptée par les gens que je documente. Parce qu'eux ils me comprennent. Alors que moi dans ma démarche autodocumentaire, je cherche à les comprendre. Dans le montage, j’arrange toutes ces voix en les écoutant d'abord, puis en les regardant pour mieux les agencer pour avoir un discours. Je me réconcilie avec eux, qu'ils soient, des génocidaires, des survivants comme moi ou des bystanders. Mon travail à moi c'est une forme de réconciliation avec les autres. Et quand j'arrive à montrer mon film, je sens que maintenant les autres m'acceptent. Ils n'ont pas rejeté mon travail. Ils ont accepté de le voir. Ils me comprennent. Depuis cinq ans, le film n’a jamais été montré dans un festival des droits de l'homme, et cela n'est pas pour m'étonner. Ces organisations des droits de l'homme auraient dû être les premières à participer à la prévention du génocide qui est au centre de ce film. Mais elles ne veulent pas montrer leurs propres échecs.

Quant à la réconciliation avec moi-même et avec Dieu, je ne l'ai pas encore atteinte. Peut-être, je n'ai pas encore eu le temps ou un espace où je peux me réconcilier avec Dieu et avec moi-même. De plus, me réconcilier avec Dieu c'est impossible 
parce que Dieu n'est pas là pour demander cet espace de réconciliation. Dieu, ce n'est pas un élément que je peux filmer. Si je veux chercher la réconciliation avec Dieu et moi-même, je vais prendre la caméra. La réconciliation exige en somme de filmer, traduire, écouter et puis voir.

A.D.-R. : Est-ce qu'il y a un travail de réconciliation à faire avec le Gilbert Ndahayo que tu as filmé durant la gacaca ? Est-ce qu'il y avait un travail de réconciliation à faire avec celui que tu as été et que tu n'es plus aujourd'hui?

G. N. : Tu veux dire les images de moi filmées durant la gacaca?

A.D.-R. : Oui, dans cette séquence il y a une certaine distance qui existe entre celui qui est filmé et celui que l'on est devenu lorsque l'on regarde celui que l'on était. Est-ce que là, il y a eu un travail où tu t’es dit : «Bien, c'était moi, j’ai dit cela, j’ai pris position de cette façon-là...» Dans des versions antérieures de Rwanda: Beyond the Deadly Pit, la scène où tu pardonnes n'a pas toujours été retenue au montage et projetée. Alors est-ce qu'il y a eu pour toi un travail de réconciliation à faire avec ce que Gilbert Ndahayo a dit en novembre 2007 lors de la gacaca pour pouvoir l'assumer et l'intégrer dans la version finale de Rwanda: Beyond a Deadly Pit en février 2012 ?

G. N. : Dans les premières versions, je n’avais pas encore accepté cette réconciliation avec les autres et surtout avec le génocidaire qui a assassiné mon père. En montrant en public le fait que j’avais pardonné et que j'étais devant la caméra, cela a eu « un effet miroir ». Je me projette en acceptant cette réconciliation pas seulement avec l'assassin que l'on voit, mais aussi avec moi acceptant la réconciliation avec les autres. Là, en premier lieu, c'était difficile d'accepter. Parce qu'il y avait cette question : « est-ce que tu t'es réconcilié pour pardonner ? » ou «Peux-tu coexister avec les assassins de tes parents? » Ça ne veut pas dire que je me suis réconcilié physiquement avec l'assassin, mais que, dans ce monde filmique ou symbolique, je me suis réconcilié avec lui. J'ai filmé et quitté ce lieu de tournage de la gacaca avec mes cassettes et ma caméra. Pour moi, il s'agissait de voir mon image à travers le miroir. Le montage reflète cette réconciliation entre moi et les autres. Il appartient aux autres de se réconcilier avec moi, en acceptant ou non mon image et celle que je donne d'eux.

Auparavant, c'est la politique qui définissait l'espace où placer le miroir ou définir ce que l'on doit raconter aux autres pour les intérêts de la réconciliation nationale. L'espace de la réconciliation avec soi n'y existe pas. Les institutions qui montrent les documentaires sur le génocide sont des tiers politiques qui tendent à censurer ce miroir de soi. Il appartient aux autres de l'accepter. Mais les multiples tiers auxquels je m’adresse ne peuvent pas m'accepter s'ils n'ont pas un espace où l'on peut se rencontrer. C'est cet espace que mon documentaire Rwanda : Beyond the Deadly Pit vise à susciter. C'est ça au fond un des problèmes de ce miroir de la réconciliation. 
A.D.-R. : Rwanda: Beyond the Deadly Pit cherche donc à créer dans l'espace public un espace de rencontre et de dialogue qui n'existait pas avant ce film?

G. N. : Oui, mais l'avènement de cet espace exige à la fois une agressivité qui vise à forger cette image en miroir de moi-même et la volonté des tiers auxquels je m'adresse à me donner un espace pour que cette réconciliation soit possible. Cet espace est disponible au sein des universités, des conférences sur le génocide et par l'entremise d'experts qui ne sont pas motivés par des intérêts politiques qui censurent les espaces publics de réconciliation. Ces instances forcent le public à me donner cet espace de réconciliation : il en va de même avec l'audience rwandaise dans la diaspora. Ma façon de me réconcilier avec les autres passe donc par la mise en dialogue des quatre tiers que j’ai représentés dans mon documentaire.

A.D.-R. : Par exemple dans des versions de Behind this Convent (2008), il y avait tous les problèmes de la réconciliation avec ton père où tu lui demandes pardon. Est-ce que là, il y a un dialogue avec un tiers posthume unique? Les dialogues que tu crées sont à la fois avec des interlocuteurs vivants, mais visent aussi à se réconcilier avec la relation que toi, héritier, tu as avec les morts ? En reprenant ce que Esther Mujawayo fait pour une de ses sœurs, Stéphanie, quand elle lui offre son témoignage comme une forme de sépulture, la dernière version de ce documentaire, Rwanda: Beyond the Deadly Pit, revêt-elle ce statut et relève-t-elle d'une forme de réconciliation posthume? Est-ce que pour toi aussi le fait de ne pas déterrer les morts - tu ne laves pas les os, tu ne portes pas le cercueil, mais tu filmes tout ceci - fait qu'un des statuts du film est de servir comme sépulture pour tes tiers posthumes? Mais une sépulture dont toi tu peux définir les formes, les modalités?

G. N. : En premier lieu, j’ai demandé pardon à mon père dans les premières versions du documentaire. C'est disons « un poème » dont les mots s'affichent sur l'écran mais pas sous une forme verbale. J'ai senti que quand j’ai accordé le pardon à la personne qui a pris la vie de mon père, que c’était là un acte de lâcheté. Même dans la création de cette œuvre, j’ai senti que j’étais un lâche. Comment aller devant les survivants du génocide et leur dire «j’ai pardonné »? Mais quand j’ai regardé l'œuvre, je me suis dit, si je l'offre en souvenir à mon père c'est là ma façon de commémorer et je crois que mon père me pardonnera ma lâcheté. Mais ça c'est une prise de conscience postérieure à la création. En ce sens, l'espace entre mon père et moi, pendant le tournage et le montage, était celui d'un acte de lâcheté.

J'ai grandi avec une grande fascination pour mon père. Je suis né dans un village dans la province du sud. Mon père était absent durant ma jeune enfance, car il étudiait le droit à l'université. C'est donc ma mère qui s'est occupée de moi jusqu’à lâge de 5 ans. Quand mon père a terminé ses études, nous avons quitté le village. À partir de ce moment-là, il a toujours pris soin de moi, beaucoup plus que des autres enfants. Dans ses derniers jours, il m’a demandé de lui pardonner son absence. Ainsi, en lui demandant de me pardonner dans le film, c'est une relation spéculaire, un 
effet miroir que je mets en place, car il a été assassiné avant que je lui accorde mon pardon. Mais en pardonnant à son assassin, je pensais trahir sa mémoire parce qu'en réalité j’ai recréé le souvenir de sa mise à mort avec des images qui n'étaient pas là. Lors de l'ouverture de la fosse mortelle, il y avait des gens qui ne savaient pas si leurs parents étaient parmi les victimes. Ils essayaient de penser qu'ils étaient là, parmi les ossements déterrés. Il y a cette négociation, cette reconnaissance et discussion à l'intérieur de soi liées au processus du deuil. Des fois, mon cœur me confortait « ne doute pas que c'est lui ». Il y a cette peur de la vérité tout comme la nécessité de savoir. Il avait toujours sa carte d'identité dans sa poche intérieure et portait ses habits. Mais avant tout, je savais qu'il était là parce que le corps de mon père était là en entier, enterré, déterré, lavé, transféré au site mémorial.

Dans ces effets de miroir, il y a le fait que déterrer mon père de l'endroit où il était constituait déjà une trahison, c'était déjà un acte de lâcheté à mes yeux. Je sentais qu'il aurait dû rester là où il était et qu'il soit là éternellement. Que l'on conserve sa mémoire là où il était sans le mettre dans un tombeau au mémorial. En premier lieu, préserver le lieu où le génocide a eu lieu, faute de quoi, il n’y a plus de signes du génocide.

Donc la mémoire est rasée et transférée dans un endroit inapproprié. La caméra suit ce transfert. À l'endroit où le génocide a eu lieu, on a rasé cette mémoire pour en faire une archive en un autre lieu. Sans la mémoire de l'endroit même où le crime du génocide a été commis, les gens risquent d'oublier ou n'ont pas le droit d'honorer cette mémoire à l'endroit où elle devrait l'être. La commémoration maintenant est transférée de l'endroit où elle devrait avoir lieu en des endroits spécifiquement désignés par une politique de la mémoire. Les activités de commémoration sont transférées dans des lieux publics où le témoignage est contrôlé. Du coup, ce témoignage ritualisé ne satisfait pas les gens qui commémorent et, à la fin, les survivants diront «j'en ai assez ». Je n'irai plus dans cet espace public, ce lieu où l'on demande aux survivants de commémorer ensemble une fois par an. Moi, je commémore chaque jour. Si l'on ne le fait pas chaque jour quand on se lève, le monde va vers l'oubli et l'oubli va amener d'autres violences.

A.D.-R. : Dans ce contexte-là, le film sert à ce moment de lieu de mémoire ou de sépulture personnalisée dans une certaine mesure puisque c'est toi qui peux définir les modalités selon lesquelles tu veux honorer tes morts?

G. N. : Si l'espace individuel a été rasé, moi je le garde sous une forme que l'on ne peut pas raser. En ce sens, je ne subis pas cette pression qui rase la mémoire parce que moi j’ai filmé ces activités au moment où le vol de la mémoire se faisait. Je crois que dans le futur, s'il y a quelque chose qui se passe, je dirai : "j’ai les preuves du vol et de l'éradication de la mémoire. » Oui, l'œuvre va rester comme sépulture et offrir un lieu de mémoire qui atteste le fait qu'il y a eu ce massacre. Visionner cela contribue dès lors à nourrir un imaginaire désireux de sauvegarder cette mémoire.

Pour revenir à la question de mon père, quand j’ai réalisé que j’ai reproduit le site 
mémoriel où il était et que j’ai conservé le lieu du massacre où il avait été tué, je me suis senti libéré de cette lâcheté. La lâcheté du pardon, la lâcheté d’être trahi par le monde et la politique de réconciliation nationale qui oblige que l'on éradique, que l'on ne commémore pas individuellement. Je me suis senti libre, parce que toujours je serai le propriétaire de cette mémoire, même si le documentaire qui est venu remplacer la fosse en l'archivant demeure, à un certain degré, une forme de lâcheté envers mon père. Lui, il est sous terre et il ne peut pas me répondre. En somme, je me suis réapproprié ce que l'on m'a volé. J'ai volé ce que l'on m'avait volé : ma mémoire et mon espace de commémoration. Toutefois, moi, je suis toujours prisonnier de moi-même. Je vis en attente d'une réconciliation avec moi-même. I 